\title{
Five year change in alcohol intake and risk of breast cancer and coronary heart disease among postmenopausal women: prospective cohort study
}

\author{
Marie K Dam, ${ }^{1}$ Ulla A Hvidtfeldt,, ${ }^{2}$ Anne Tjønneland, ${ }^{3}$ Kim Overvad,, 45 Morten Grønbæk, ${ }^{1}$ \\ Janne S Tolstrup ${ }^{1}$
}

${ }^{1}$ National Institute of Public Health, University of Southern Denmark, 1353 Copenhagen K, Denmark

${ }^{2}$ Social Medicine Section, Department of Public Health, University of Copenhagen, Copenhagen

${ }^{3}$ Danish Cancer Society Research Centre, Copenhagen

${ }^{4}$ Department of Public Health, Department of Epidemiology, Aarhus University, Aarhus, Denmark

${ }^{5}$ Department of Cardiology, Aalborg University Hospital, Hobrogade, Aalborg, Denmark Correspondence to: J S Tolstrup jst@niph.dk Additional material is published online only. To view please visit the journal online.

Cite this as: $B M$ J 2016;353:i2314 http://dx.doi.org/10.1136/bmj.i2314 Accepted: 12 April 2016

\section{ABSTRACT}

\section{OBJECTIVE}

To test the hypothesis that postmenopausal women who increase their alcohol intake over a five year period have a higher risk of breast cancer and a lower risk of coronary heart disease compared with stable alcohol intake.

DESIGN

Prospective cohort study.

SETTING

Denmark, 1993-2012.

\section{PARTICIPANTS}

21523 postmenopausal women who participated in the Diet, Cancer, and Health Study in two consecutive examinations in 1993-98 and 1999-2003. Information on alcohol intake was obtained from questionnaires completed by participants.

\section{MAIN OUTCOME MEASURES}

Incidence of breast cancer, coronary heart disease, and all cause mortality during 11 years of follow-up. Information was obtained from the Danish Cancer Register, Danish Hospital Discharge Register, Danish Register of Causes of Death, and National Central Person Register. We estimated hazard ratios according to five year change in alcohol intake using Cox proportional hazards models.

RESULTS

During the study, 1054, 1750, and 2080 cases of breast cancer, coronary heart disease, and mortality occurred, respectively. Analyses modelling five year change in alcohol intake with cubic splines showed that women who increased their alcohol intake over the five year period had a higher risk of breast cancer and a lower risk of coronary heart disease than women

\section{WHAT IS ALREADY KNOWN ON THIS TOPIC}

Many studies have shown that alcohol intake is associated with increased risk of breast cancer and decreased risk of coronary heart disease

Most evidence consists of observational studies, correlating alcohol intake measured at one point in time with disease incidence

Little is known about the effect of a change in alcohol intake on the risk of breast cancer and coronary heart disease

\section{WHAT THIS STUDY ADDS}

We found that an increased alcohol intake over a five year period resulted in a higher risk of breast cancer and a lower risk of coronary heart disease among postmenopausal women, compared with a stable alcohol intake

Results support the hypotheses that alcohol is associated with breast cancer and coronary heart disease in opposite directions

with a stable alcohol intake. For instance, women who increased their alcohol intake by seven or 14 drinks per week (corresponding to one or two drinks more per day) had hazard ratios of breast cancer of $1.13(95 \%$ confidence interval 1.03 to 1.23 ) and 1.29 (1.07 to 1.55), respectively, compared to women with stable intake, and adjusted for age, education, body mass index, smoking, Mediterranean diet score, parity, number of births, and hormone replacement therapy. For coronary heart disease, corresponding hazard ratios were 0.89 ( 0.81 to 0.97 ) and 0.78 (0.64 to 0.95 ), respectively, adjusted for age, education, body mass index, Mediterranean diet score, smoking, physical activity, hypertension, elevated cholesterol, and diabetes. Results among women who reduced their alcohol intake over the five year period were not significantly associated with risk of breast cancer or coronary heart disease. Analyses of all cause mortality showed that women who increased their alcohol intake from a high intake ( $\geq 14$ drinks per week) to an even higher intake had a higher mortality risk that women with a stable high intake.

\section{CONCLUSION}

In this study of postmenopausal women over a five year period, results support the hypotheses that alcohol intake is associated with increased risk of breast cancer and decreased risk of coronary heart disease.

\section{Introduction}

The effects of alcohol on the risk of breast cancer and coronary heart disease, two major diseases worldwide, have been thoroughly investigated over past decades and seem to be opposite. Studies have shown a direct relation between alcohol consumption and breast cancer, ${ }^{1-5}$ whereas the observed risk for coronary heart disease is lower among light to moderate drinkers than among abstainers. ${ }^{67}$ Plausible biological mechanisms have been suggested for breast cancer ${ }^{2389}$ and coronary heart disease, ${ }^{10-13}$ which speak in favour of the causality of these findings. Regarding the association between alcohol intake and all cause mortality, studies have shown a U or J shaped association, suggesting that light to moderate alcohol intake has a protective effect, whereas both abstainers and heavy drinkers are at an increased risk. ${ }^{14-16}$

Most evidence is obtained from observational studies that correlate alcohol intake measured at one time point to the following disease incidence. Such results could be biased by confounding or by sick quitting (that is, individuals who give up drinking for reasons of ill health and are thus at higher risk). ${ }^{101117}$ Long 
term randomised trials providing important experimental evidence could in principle offer the final answer, but owing to ethical and logistical reasons, such studies may never be conducted. Another step toward building on the evidence that alcohol is or is not a causal factor for breast cancer (increasing risk) and coronary heart disease (decreasing risk) is to examine whether a change in alcohol intake, defined by the difference in alcohol intake measured at two time points, results in a subsequent altered risk in hypothesised directions. ${ }^{18}$ Thus, in this study, we compare changing and stable patterns of alcohol intake during a five year period in a prospectively followed cohort of postmenopausal women. We aimed to test the hypotheses that increased alcohol intake is associated with a higher risk of breast cancer and a lower risk of coronary heart disease compared with stable alcohol intake (and vice versa).

\section{Methods \\ Study population}

From 1993 to 1997, 79729 women and 80996 men, aged 50-64 years, were invited to participate in the Diet, Cancer, and Health Study, a population cohort study. Eligible cohort members were born in Denmark, lived in the greater Copenhagen or Aarhus area, and had no previous cancers. ${ }^{19}$ Overall, 29535 (37\%) women agreed to participate in a study examination in 1993-98 and, of these, 23796 (81\%) agreed to participate again in a second examination about five years later in 1999-2003.

We excluded 2273 premenopausal women (defined as women who, in the 1999-2003 examination, reported having had menstruations during the past year). The final study population for analyses of all cause mortality included 21523 women. Women who were registered with a diagnosis of breast cancer $(n=423)$ or coronary heart disease $(n=884)$ at baseline (1999-2003) were excluded from analyses of breast cancer and coronary heart disease, respectively.

\section{Alcohol intake}

A detailed food frequency questionnaire consisting of 192 items was enclosed with the invitation to participate. ${ }^{20}$ This questionnaire was checked by an interviewer during a clinic visit, when another questionnaire concerning lifestyle and background factors was completed. In the food frequency questionnaire, alcohol intake was reported as the average units of beer, wine, and spirits consumed over the preceding year. Total intake was calculated and converted into number of standard drinks, defined as containing $12 \mathrm{~g}$ of ethanol.

\section{Covariates}

Analyses were adjusted for known risk factors for breast cancer, coronary heart disease, and mortality. All estimates were adjusted for school education $(<8,8-10$, and $\geq 11$ years of school), body mass index (continuous), smoking (entered as two variables: a variable categorised as never, past, and current smoker; and a continuous variable for the number of grams of tobacco smoked per day), and the Mediterranean diet score (continuous). ${ }^{202122}$ Estimates for breast cancer were further adjusted for parity (parous or nulliparous) and number of births (continuous), and for use of hormone replacement therapy (never, past, and current). The estimates for coronary heart disease and mortality were further adjusted for physical activity (three indicator variables for performing the following activities weekly in leisure time: taking walks, bicycling, and undertaking sports) as well as baseline hypertension, elevated cholesterol, and diabetes. Finally, the analyses of mortality were also adjusted for self-reported health status (entered as a continuous variable consisting of five levels of health status, ranging from level one being excellent to level five being poor).

\section{Information on breast cancer, coronary heart disease, and all cause mortality}

Information on breast cancer was obtained from the Danish Cancer Register, ${ }^{2324}$ which contains data on all cancer patients in Denmark. Information on coronary heart disease was obtained from the Danish Hospital Discharge Register ${ }^{25}$ and from the Danish Register of Causes of Death, ${ }^{2627}$ which contain information on hospital admissions and causes of death in Denmark, respectively. In all three registers, diagnoses are classified according to the international classification of diseases (ICD). The relevant ICD-10 code for breast cancer is $\mathrm{C} 50$. The relevant ICD-8 codes for coronary heart disease are 410-414, and the ICD-10 codes are I20-I25. The exact diagnosis for breast cancer has been validated, ${ }^{28}$ and the validity of the Danish Hospital Discharge Register is generally considered to be high. ${ }^{29}$ The registers were updated until 31 December 2012.

Information on vital status (including death) of the participants was obtained from the National Central Person Register. Participants were followed from baseline in 1999-2003 until the date of outcome of interest (breast cancer, coronary heart disease, or death), emigration, or end of follow-up on 31 December 2012, whichever came first.

\section{Statistical analysis}

Analyses were performed using the Stata program software version 14. To account for missing values, we used multiple imputation by chained equations. ${ }^{30}$ For each outcome (breast cancer, coronary heart disease, and mortality), imputation models were run separately, resulting in three datasets, each with 20 repetitions. Potential confounders were imputed. Each imputation model included variables that were hypothesised to predict missing information (age, education, smoking, health status (hypertension, elevated cholesterol, and diabetes)) and the respective outcome. Following imputation, the principle for data analysis was that estimation was performed on each imputation separately and then combined using Rubin's rules. ${ }^{31}$ The number of missing values was nine (school education), 67 (body mass index), one (parity), 246 (hormone replacement therapy), 202 (taking walks), 123 (bicycling), 207 (undertaking sports), and 161 (self-reported health status). Thus, the number 
of missing was low and a maximum of $1.1 \%$ (for hormone replacement therapy).

We calculated risk estimates by using Cox proportional hazards models with delayed entry. Age (in days) was used as the underlying time axis to ensure maximal adjustment for confounding by age. We examined the Cox proportional hazards assumption by plots of $\log (-$ time) against $\log (-\log ($ survival probability)) and statistically by introducing interaction terms between age and change in alcohol intake in the model, with no violations detected.

Firstly, we examined main associations between alcohol intake at baseline (1999-2003) and risk of breast cancer, coronary heart disease, and all cause mortality in separate analyses using alcohol intake (drinks per week) in six categories to verify that the results were as expected. To test for U shape, alcohol was entered as a linear and a squared variable. The $\mathrm{P}$ value for the squared term denotes $\mathrm{P}$ for $\mathrm{U}$ shape.

We then examined the association between combinations of alcohol intake at the examinations in 1993-98 and 1999-2003 (mean time 5.4 years (range 3.2-8.5)) and the subsequent risk of breast cancer, coronary heart disease, and all cause mortality. We used all possible combinations of alcohol intake in the two examinations $(<7$, $7-13$, and $\geq 14$ drinks per week), which yielded nine combinations of intake. Owing to a higher statistical power in the analysis of mortality, changes in alcohol intake here were defined by the possible combinations of alcohol intake in the two examinations $(<7,7-13,14-20$, and $\geq 21$ drinks per week), which yielded 16 combinations of intake. A minimum change of one drink per week was required in order to change from one category of alcohol intake in the 1993-98 examination to another in the 1999-2003 examination. Results from this analysis were presented with the reference defined by women with stable alcohol intake for each level of alcohol intake in the 1993-98 examination, and by stable light-drinkers ( $<7$ drinks per week in both examinations), respectively.

Furthermore, we calculated the five year change in alcohol intake by subtracting the intake at the 1993-98 examination from the intake at the 1999-2003 examination. Hence, a negative value represented a decrease in alcohol intake, while a positive value represented an increase. This variable was divided into seven categories $(<-14,-14$ to $<-7,-7$ to $<-1,-1$ to $<1,1$ to $6.9,7$ to 13.9 , and $\geq 14$ drinks per week). This analysis was carried out for breast cancer and coronary heart disease, with women with stable alcohol intake (change of -1 to $<1$ drink per week) as the reference. Change in alcohol intake was also modelled continuously by cubic splines to account for non-linear associations. ${ }^{3233}$ To avoid outliers to influence results inexpediently, women who changed their alcohol intake outside the $0.5-99.5 \%$ percentiles of the distribution of change in alcohol intake were excluded (210 and 206 women for the analysis of breast cancer and coronary heart disease, respectively).

An underlying assumption in such analyses is that the risk is independent of the starting point in the 199398 examination and only depends on the change, meaning that the risk of disease increases or decreases equally by a given change in alcohol intake. To test this assumption, we tested for interaction between change in alcohol intake and alcohol intake at the 1993-98 examination. For this purpose, cross products between the five year change in alcohol intake in seven categories and alcohol intake in the 1993-98 examination continuously were entered in a model together with the main effects. However, because it was expected that a high alcohol intake in the 1993-98 examination could influence the subsequent risk of disease, despite a decrease in intake, the analyses were adjusted for alcohol intake in the 1993-98 examination. We also repeated analyses without adjusting for alcohol intake in the 1993-98 examination.

Finally, we did several sensitivity analyses for breast cancer, coronary heart disease, and all cause mortality. These consisted of analyses that each omitted the first three years of follow-up (to reduce risk bias from sick quitters), excluded abstainers (women who did not drink any alcohol in either examination), and excluded all missing values. To take into account competing risks in analyses of breast cancer and coronary heart disease (that is, the fact that some women died during follow-up before potentially having the disease in question), we performed analyses of breast cancer and coronary heart disease by adjusting for competing risks. Around $9 \%$ of the women died during follow-up.

For breast cancer, additional sensitivity analyses excluded women with a history of benign breast tumour surgery before baseline, adjusted for region (town), adjusted for changes in body mass index and use of hormone replacement therapy between the examinations in 1993-98 and 1999-2003, and tested for interaction with hormone replacement therapy. For coronary heart disease, additional sensitivity analyses excluded women with any type of cardiovascular disease before baseline; omitted adjustments for hypertension, elevated cholesterol, and diabetes; adjusted for changes in body mass index, smoking habits, hypertension, elevated cholesterol, and diabetes between the examinations in 1993-98 and 1999-2003; and tested for interaction with smoking. Finally, we repeated analyses for coronary heart disease, restricted to the diagnosis of myocardial infarction (ICD-8 code 410 and ICD-10 codes I21-I22) as the outcome. Regarding mortality, another sensitivity analysis adjusted for changes in body mass index, smoking habits, hypertension, elevated cholesterol, and diabetes between study examinations.

\section{Patient involvement}

No patients were involved in setting the research question or the outcome measures, nor were they involved in developing plans for recruitment, design, or implementation of the study. No patients were asked to advise on interpretation or writing up of results. There are no plans to disseminate the results of the research to study participants or the relevant patient community.

\section{Results}

Mean follow-up time was 10.8 years for breast cancer, 10.6 years for coronary heart disease, and 11.0 years for 


\begin{tabular}{|c|c|c|c|c|c|c|c|}
\hline & \multicolumn{2}{|l|}{ All women } & \multicolumn{5}{|c|}{ Change in alcohol intake (no of drinks/week)†‡ } \\
\hline & \multirow{2}{*}{$\begin{array}{l}\text { Complete } \\
\text { datat }\end{array}$} & \multirow{2}{*}{$\begin{array}{l}\text { Imputed } \\
\text { datat }\end{array}$} & \multicolumn{2}{|l|}{ Decreased intake } & \multirow{2}{*}{$\begin{array}{l}\text { Stable intake } \\
-1 \text { to } 1\end{array}$} & \multicolumn{2}{|l|}{ Increased intake } \\
\hline & & & $<-7$ & -7 to $<-1$ & & 1 to 6 & $\geq 7$ \\
\hline Total & $20599(96)$ & $21523(100)$ & $1487(7)$ & $5508(27)$ & $7422(36)$ & $4227(21)$ & $1955(9)$ \\
\hline Age (years; mean (range)) & $62.2(53.6-72.9)$ & $62.2(53.6-72.9)$ & $62.2(53.8-71.1)$ & $62.0(54.1-72.9)$ & $62.3(53.6-72.0)$ & $62.4(54.2-71.6)$ & $62.1(54.2-71.8)$ \\
\hline $\begin{array}{l}\text { Alcohol intake (drinks/week } \neq \S ; \\
\text { median (5-95\% range) }\end{array}$ & $4.9(0.0-25.2)$ & $4.8(0.0-25.2)$ & $7.8(1.0-24.7)$ & $3.4(0.6-19.6)$ & $1.8(0.0-18.9)$ & $7.6(2.1-24.4)$ & $20.3(12.6-49.7)$ \\
\hline School education $<8$ years & $6211(30)$ & $6551(30)$ & $377(25)$ & $1612(29)$ & $2577(35)$ & $1195(28)$ & $450(23)$ \\
\hline $\begin{array}{l}\text { Body mass index (median (5-95\% } \\
\text { range)) }\end{array}$ & $24.7(19.8-33.5)$ & $24.7(19.8-33.6)$ & $24.3(19.4-32.9 .0)$ & $24.8(19.9-33.3)$ & $24.9(19.7-34.8)$ & $24.5(19.8-32.5)$ & $24.3(19.6-32.0)$ \\
\hline $\begin{array}{l}\text { Mediterranean diet score (median } \\
(5-95 \% \text { range) }\end{array}$ & $5(3-7)$ & $5(3-7)$ & $5(3-7)$ & $5(3-7)$ & $5(2-7)$ & $5(3-8)$ & $5(2-7)$ \\
\hline Nulliparous & $2447(12)$ & $2543(12)$ & $222(15)$ & $595(11)$ & $876(12)$ & $517(12)$ & $237(12)$ \\
\hline Births 1 (median (5-95\% range)) & $2(1-4)$ & $2(1-4)$ & $2(1-4)$ & $2(1-4)$ & $2(1-4)$ & $2(1-4)$ & $2(1-4)$ \\
\hline $\begin{array}{l}\text { Hormone replacement therapy, } \\
\text { current users }\end{array}$ & $5528(27)$ & $5740(27)$ & $401(27)$ & $1538(28)$ & $1954(26)$ & $1107(26)$ & $528(27)$ \\
\hline Current smokers & $5203(25)$ & $5450(25)$ & $526(35)$ & $1321(24)$ & $1889(25)$ & $920(22)$ & $547(28)$ \\
\hline Physically inactive ${ }^{\star \star}$ & $2293(11)$ & $2350(11)$ & $209(14)$ & & $904(12)$ & $385(9)$ & $207(11)$ \\
\hline Hypertensiont† & $5323(26)$ & $5553(26)$ & $395(27)$ & $1397(25)$ & $1934(26)$ & $1088(26)$ & $509(26)$ \\
\hline Elevated cholesterolt† & $4321(21)$ & $4531(21)$ & $309(21)$ & $1087(20)$ & $1590(21)$ & $924(22)$ & $411(21)$ \\
\hline Diabetest† & $595(3)$ & $634(3)$ & $44(3)$ & $152(3)$ & $275(4)$ & $89(2)$ & $35(2)$ \\
\hline Poor self-rated health $\neq \ddagger$ & $2941(14)$ & $3092(14)$ & $228(15)$ & $775(14)$ & $1246(17)$ & $467(11)$ & $225(12)$ \\
\hline \multicolumn{8}{|l|}{ 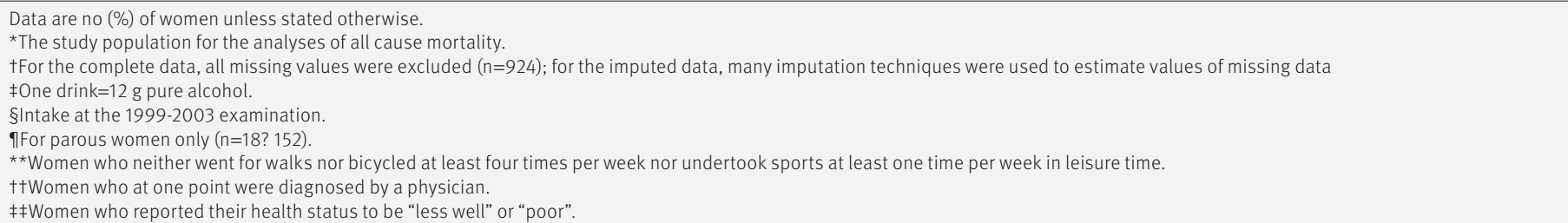 } \\
\hline
\end{tabular}

all cause mortality (range for all outcomes 0.0-13.3 years). During follow-up, 1054 cases of breast cancer and 1750 cases of coronary heart disease occurred, and 2080 women died.

\section{Baseline characteristics}

Among the 21523 postmenopausal women included in the mortality analyses, the median alcohol intake was 5.4 (5-95\% range 0.3-23.6) and 4.8 (0.0-25.2) drinks per week in the 1993-98 and 1999-2003 examinations, respectively. The median five year change in alcohol intake was 0.0 (-10 to 12) drinks per week. In 1993-98, 518 (2\%) of women were abstainers, and in 1999-2003, this number was 1115 (5\%).

Baseline characteristics can be seen in table 1. Mean age at baseline was 62 years and $36 \%$ of the women had a stable alcohol intake. In general, most covariates were equally distributed by change in alcohol intake. However, the proportion of women with a school education of shorter than eight years was highest among stable drinkers (35\%), and the highest proportion of current smokers was found among women who decreased their alcohol intake by more than seven drinks per week (35\%). This group also had the largest proportion of physically inactive women (14\%).

Baseline alcohol intake and risk of breast cancer, coronary heart disease, and all cause mortality Table 2 presents the main associations between alcohol intake at baseline (1999-2003) and risk of breast cancer, coronary heart disease, and all cause mortality among study participants. As expected, a higher risk of breast cancer, a lower risk of coronary heart disease, and a U shaped association with mortality were observed with increasing alcohol intake.

\section{Breast cancer}

Table 3 shows hazard ratios of breast cancer for nine combinations of alcohol intake in the 1993-98 and 19992003 examinations. In general, results showed that postmenopausal women who increased their alcohol intake over the five year period had a subsequent higher risk of breast cancer than women with stable alcohol intake. In both examinations, women who increased their alcohol intake had raised risks for breast cancer ( $<7$ to $7-13$ drinks/week: hazard ratio 1.38 (95\% confidence interval 1.10 to 1.73 ); $<7$ to $\geq 14: 1.16$ (0.81 to 1.67 ); 7-13 to $\geq 14: 1.44$ (1.12 to 1.86 )) compared with women drinking fewer than seven drinks per week. For women who reduced their alcohol intake over the five year period, none of the hazard ratios was significantly associated with breast cancer.

Figure 1 shows hazard ratios for breast cancer among postmenopausal women by five year change in alcohol intake with stable alcohol intake as reference, modelled as cubic splines (blue solid curve) and categories (stepped line). Women with increased alcohol intake had a subsequent higher risk of breast cancer compared to women with a stable alcohol intake. For instance, hazard ratios among women who increased their alcohol intake by seven and 14 drinks per week were 1.13 (95\% confidence interval 1.03 to 1.23 ) and 1.29 (1.07 to 
Table 2 | Risk of breast cancer, coronary heart disease, and all cause mortality among postmenopausal women by alcohol intake in the $1999-2003$ examination

\begin{tabular}{|c|c|c|c|c|c|c|c|}
\hline & \multicolumn{6}{|c|}{ Alcohol intake (drinks/week)* } & \multirow{2}{*}{ P for trend } \\
\hline & $<1$ & $1-6$ & $7-13$ & $14-20$ & $21-27$ & $\geq 28$ & \\
\hline \multicolumn{8}{|l|}{ Breast cancert } \\
\hline No of cases/person years & $144 / 33242$ & $440 / 104462$ & $233 / 44600$ & $133 / 26801$ & $50 / 9629$ & $54 / 8468$ & \multirow{2}{*}{0.0079} \\
\hline Hazard ratio $(95 \% \mathrm{Cl})$ & 0.99 (0.82 to 1.20$)$ & 1.00 & 1.27 (1.08 to 1.49$)$ & 1.14 (0.94 to 1.39) & 1.18 (0.88 to 1.58$)$ & 1.45 (1.09 to 1.93$)$ & \\
\hline \multicolumn{8}{|l|}{ Coronary heart disease } \\
\hline No of cases/person years & $334 / 30947$ & $795 / 100312$ & $332 / 43539$ & $170 / 26289$ & $60 / 9634$ & $59 / 8308$ & \multirow{2}{*}{0.0019} \\
\hline Hazard ratio $(95 \% \mathrm{Cl})$ & 1.16 (1.02 to 1.32$)$ & 1.00 & $1.00(0.88$ to 1.14$)$ & 0.81 (0.69 to 0.96$)$ & $0.77(0.59$ to 1.00$)$ & 0.83 (0.63 to 1.08) & \\
\hline \multicolumn{8}{|l|}{ All cause mortality§ } \\
\hline No of cases/person years & $436 / 34373$ & $885 / 108511$ & $340 / 46642$ & $212 / 28107$ & $103 / 10147$ & $104 / 8939$ & \multirow{2}{*}{$0.004^{\star *}$} \\
\hline Hazard ratio $(95 \% \mathrm{Cl})$ & 1.11 (0.98 to 1.25$)$ & 1.00 & $0.96(0.85$ to 1.10$)$ & 0.89 (0.76 to 1.03$)$ & 1.16 (0.94 to 1.42) & 1.21 (0.98 to 1.49) & \\
\hline \multicolumn{8}{|c|}{ 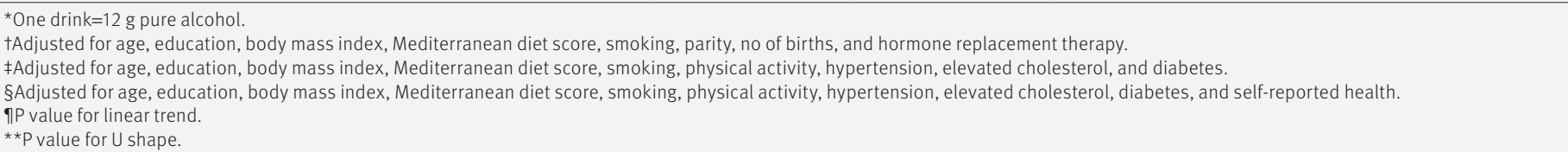 } \\
\hline
\end{tabular}

1.55), respectively. For women who decreased their alcohol intake, the tendency in results was unclear, and results were not significant. The spline curve fitted the categorical analysis well for categories in the middle of the range ( -14 to $<-7,-7$ to $<-1,-1$ to $<1,1$ to 6.9 , and 7 to 13.9). However, in the extremes and especially for the category of at least 14 drinks per week, the spline curve and hazard ratio as estimated by categorical analysis diverged, most likely due to low statistical power. The interaction test between alcohol intake in the 1993-98 examination and change in alcohol intake was not significant $(\mathrm{P}=0.88)$. Repeating the analysis without adjusting for alcohol intake in the 1993-98 examination did not change results.

In the sensitivity analyses, we excluded women with a history of benign breast tumour surgery before baseline $(\mathrm{n}=2867)$. However, this exclusion did not change the estimates substantially (data not shown). To

Table 3 | No of cases and person years, and hazard ratios (95\% confidence intervals) of breast cancer among postmenopausal women by alcohol intake in 1993-98 and 1999-2003 examinations

\begin{tabular}{|c|c|c|c|}
\hline \multirow{2}{*}{$\begin{array}{l}\text { 1993-98 examination } \\
\text { (drinks/week)* }\end{array}$} & \multicolumn{3}{|c|}{ 1999-2003 examination (drinks/week)* } \\
\hline & $<>$ & $7-13$ & $\geq 14$ \\
\hline \multicolumn{4}{|c|}{ No of cases/person years } \\
\hline$<7$ & 496/119113† & $90 / 16354$ & $32 / 6434$ \\
\hline $7-13$ & $66 / 15051$ & $99 / 20319+$ & $69 / 11368$ \\
\hline$\geq 14$ & $26 / 4192$ & $40 / 7285$ & $136 / 27086 t$ \\
\hline \multicolumn{4}{|c|}{ Stratified analysis (hazard ratio $(95 \% \mathrm{Cl})) \neq$} \\
\hline$<7$ & $1.00 t$ & 1.38 (1.10 to 1.73 ) & 1.16 (0.81 to 1.67$)$ \\
\hline $7-13$ & 0.88 (0.64 to 1.20$)$ & $1.00 t$ & 1.18 (0.87 to 1.62$)$ \\
\hline$\geq 14$ & 1.23 (0.81 to 1.88$)$ & 1.16 (0.81 to 1.66$)$ & $1.00+$ \\
\hline \multicolumn{4}{|c|}{ Joint category analysis (hazard ratio $(95 \% \mathrm{Cl})) \S$} \\
\hline$<7$ & $1.00+$ & 1.38 (1.10 to 1.73$)$ & 1.16 (0.81 to 1.67$)$ \\
\hline $7-13$ & 1.07 (0.83 to 1.38$)$ & $1.22(0.98$ to 1.52$) \dagger$ & $1.44(1.12$ to 1.86$)$ \\
\hline$\geq 14$ & 1.44 (0.97 to 2.13$)$ & 1.35 (0.98 to 1.87$)$ & $1.16(0.96$ to 1.41$) \dagger$ \\
\hline
\end{tabular}

Estimates are adjusted for age, education, body mass index, smoking, Mediterranean diet score, parity, no of births, and hormone replacement therapy.

*Alcohol intake recorded by each examination. One drink=12 g pure alcohol.

tStable alcohol intake, shown as diagonal line of cells in each section; cells below this diagonal line indicate

decreased intake, cells above the line indicate increased intake.

¥Stratified by alcohol intake in the 1993-98 examination, with stable alcohol intaket as reference.

§loint category analysis, with the reference group as stable alcohol intake of fewer than seven drinks per week in

the 1993-98 and 1999-2003 examinations. explore whether the relatively high risk of breast cancer among women who decreased their alcohol intake was due to sick quitters, we performed an analysis omitting the first three years of follow-up. This analysis did not affect results. Excluding abstainers ( $\mathrm{n}=382$ ) did not cause a change in estimates (data not shown), nor did excluding all of the originally missing values ( $n=312$; data not shown). We repeated analyses taking into account competing risks from death, which caused changes in estimates of a maximum of $2 \%$ (data not shown). Adjusting analyses for smoking and region did not change the estimates (data not shown), nor did adjusting for changes in body mass index and use of hormone replacement therapy between the two

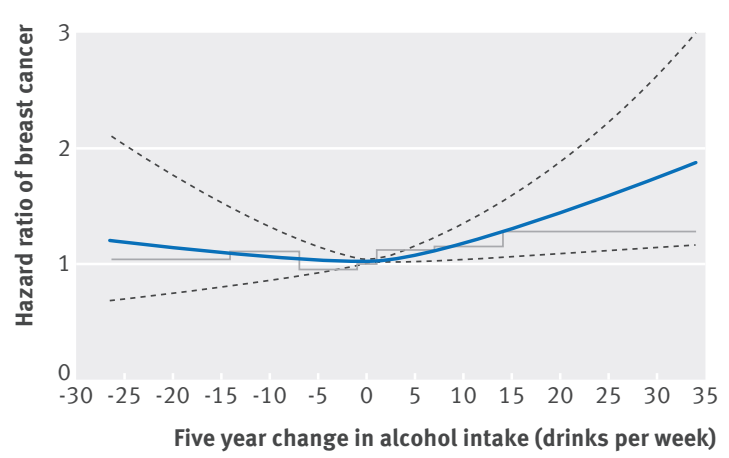

Fig 1 | Hazard ratios of breast cancer among postmenopausal women by five year change in alcohol intake. Blue solid curve (black dashed lines)=hazard ratios ( $95 \%$ confidence intervals) of breast cancer by change in alcohol intake from the 1993-98 to the 1999-2003 examinations, modelled by cubic splines; stepped line=hazard ratios of breast cancer for seven categories of change in alcohol intake. Women who changed their alcohol intake outside the $0.5-99.5 \%$ percentiles of the distribution of change in alcohol intake were excluded from analyses presented in the figure, resulting in 20890 women and 1045 cases of breast cancer eligible for analysis. Estimates are adjusted for age, education, body mass index, smoking, Mediterranean diet score, parity, number of births, hormone replacement therapy, and alcohol intake in the 1993-98 examination 
study examinations (data not shown). The test for interaction between change in alcohol intake and use of hormone replacement therapy was not significant $(\mathrm{P}=0.34)$.

\section{Coronary heart disease}

Table 4 shows hazard ratios of coronary heart disease for nine combinations of alcohol intake in the 1993-98 and 1999-2003 examinations. In general, results showed a tendency that postmenopausal women who increased their alcohol intake over the five year period had a subsequent lower risk of coronary heart disease than women with stable alcohol intake. Women with increased alcohol intake had hazard ratios for coronary heart disease of 0.88 (95\% confidence interval 0.72 to $1.07 ;<7$ to $7-13$ drinks per week), 0.81 ( 0.60 to 1.10 ; $<7$ to $\geq 14$ ), and 0.65 ( 0.50 to $0.84 ; 7-13$ to $\geq 14$ ), compared with women drinking fewer than seven drinks per week in both examinations.. For women who decreased their alcohol intake between the two examinations, results were more ambiguous and all not significant.

Figure 2 shows the hazard of coronary heart disease among postmenopausal women by five year change in alcohol intake with stable alcohol intake as reference, modelled as cubic splines (blue solid curve) and categories (stepped line). The spline curve fitted the categorical analysis well. This analysis also showed that women who increased their alcohol intake between the two examinations had a lower risk of coronary heart disease than women with stable intake. For instance, women who increased their alcohol intake by seven or 14 drinks per week had hazard ratio of 0.89 (95\% confidence interval 0.81 to 0.97 ) and 0.78 (0.64 to 0.95), respectively. For women who decreased their alcohol intake, results were not significant. The test for interaction between alcohol intake in the 1993-98 examination

\begin{tabular}{|c|c|c|c|}
\hline \multirow{2}{*}{$\begin{array}{l}\text { 1993-98 examination } \\
\text { (drinks/week)* }\end{array}$} & \multicolumn{3}{|c|}{ 1999-2003 examination (drinks/week)* } \\
\hline & $<7$ & $7-13$ & $\geq 14$ \\
\hline \multicolumn{4}{|c|}{ No of cases/person years } \\
\hline$<7$ & $1003 / 113100 \dagger$ & $114 / 15894$ & $45 / 6269$ \\
\hline $7-13$ & $101 / 14738$ & 151/19769† & $62 / 11272$ \\
\hline$\geq 14$ & $38 / 4057$ & $53 / 7254$ & $183 / 26675 t$ \\
\hline \multicolumn{4}{|c|}{ Stratified analysis (hazard ratio $(95 \% \mathrm{CI})) \neq$} \\
\hline$<7$ & $1.00+$ & $0.88(0.72$ to 1.07$)$ & $0.81(0.60$ to 1.10$)$ \\
\hline 7-13 & $0.86(0.67$ to 1.10$)$ & $1.00 \dagger$ & 0.67 (0.50 to 0.91) \\
\hline$\geq 14$ & $1.29(0.91$ to 1.84$)$ & 1.11 (0.81 to 1.51) & $1.00+$ \\
\hline \multicolumn{4}{|c|}{ Joint category analysis (hazard ratio $(95 \% \mathrm{CI})) \S$} \\
\hline$<7$ & $1.00 \dagger$ & 0.88 (0.72 to 1.07$)$ & $0.81(0.60$ to 1.10$)$ \\
\hline $7-13$ & 0.83 (0.67 to 1.02) & $0.96(0.81$ to 1.15$) \dagger$ & $0.65(0.50$ to 0.84$)$ \\
\hline$\geq 14$ & $1.02(0.73$ to 1.41$)$ & $0.87(0.66$ to 1.15$)$ & $0.79(0.67$ to 0.92$) \dagger$ \\
\hline \multicolumn{4}{|c|}{$\begin{array}{l}\text { Estimates are adjusted for age, education, body mass index, Mediterranean diet score, smoking, physical } \\
\text { activity, hypertension, elevated cholesterol, and diabetes. } \\
\text { *Alcohol intake recorded by each examination. One drink=12 g pure alcohol. } \\
\text { tStable alcohol intake, shown as diagonal line of cells in each section; cells below this diagonal line indicate } \\
\text { decreased intake, cells above the line indicate increased intake. } \\
\text { fStratified by alcohol intake in the } 1993-98 \text { examination, with stable alcohol intaket as reference. } \\
\text { §Joint category analysis, with the reference group as stable alcohol intake of fewer than seven drinks per week in } \\
\text { the } 1993-98 \text { and } 1999-2003 \text { examinations. }\end{array}$} \\
\hline
\end{tabular}

Table 4 | No of cases and person years, and hazard ratios ( $95 \%$ confidence intervals) of and 1999-2003 examination

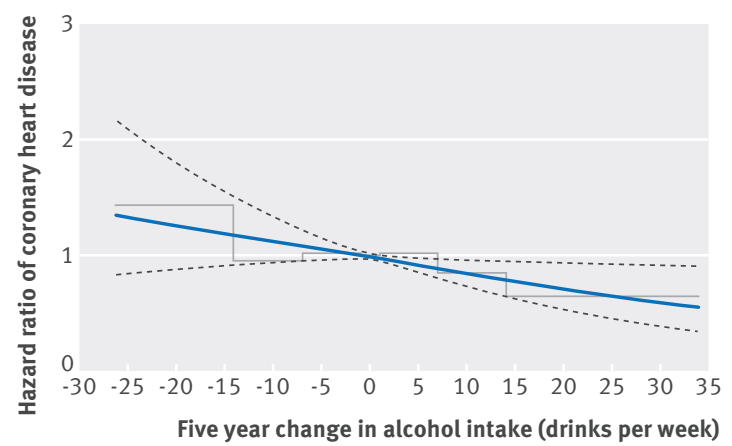

Fig 2 | Hazard ratios of coronary heart disease among postmenopausal women by five year change in alcohol intake. Blue solid curve (black dashed lines)=hazard ratios ( $95 \%$ confidence intervals) of coronary heart disease by change in alcohol intake from the 1993-98 to the 19992003 examinations, modelled by cubic splines; stepped line=hazard ratios of coronary heart disease for seven categories of change in alcohol intake. Women who changed their alcohol intake outside the 0.5-99.5\% percentiles of the distribution of change in alcohol intake were excluded from analyses presented in the figure, resulting in 20433 women and 1730 cases of coronary heart disease eligible for analysis. Estimates are adjusted for age, education, body mass index, Mediterranean diet score, smoking, physical activity, hypertension, elevated cholesterol, diabetes, and alcohol intake in the 1993-98 examination

and change in alcohol intake was also not significant $(\mathrm{P}=0.18)$. Repeating the analysis without adjusting for alcohol intake in the 1993-98 examination did not change results.

Several sensitivity analyses were performed, which did the following:

- Excluded women with any type of cardiovascular disease before baseline $(n=188)$

- Omitted the first three years of follow-up

- Excluded abstainers ( $\mathrm{n}=358)$

- Excluded all originally missing values $(n=506)$

- Omitted adjustments for hypertension, elevated cholesterol, and diabetes

- Adjusted for changes in body mass index, smoking habits, hypertension, elevated cholesterol, and diabetes between the two study examinations.

All of these analyses caused minor changes in the results only (data not shown). Taking into account competing risks also did not cause changes in results (changes in estimates $<2 \%$, data not shown). A test for interaction with smoking was not significant $(\mathrm{P}=0.91)$. Finally, by repeating the analyses restricting the outcome to myocardial infarction only (483 cases), the tendency in results remained, but confidence intervals were wider. However, the overall test for the spline curve remained significant $(\mathrm{P}=0.02)$. Nonetheless, because the power of these analyses was limited, we used the broader definition of coronary heart disease as the outcome in the main analyses, being conscious that the validity of this diagnosis as an entity is lower. ${ }^{34}$ 


\section{All cause mortality}

The web table lists hazard ratios of all cause mortality among postmenopausal women for 16 combinations of alcohol intake in the 1993-98 and 1999-2003 examinations. In general, results indicated that women who changed their alcohol intake from a moderate or high intake (that is, $7-13$ or 14-20 drinks per week) to a low or heavy intake (that is, $<7$ or $\geq 21$ drinks per week) had a higher mortality risk compared with women with a stable moderate or high intake. However, confidence intervals were generally wide. We did sensitivity analyses similar to those performed for coronary heart disease, but these did not lead to results different from those presented in the web table.

The interaction test between alcohol intake in the 1993-98 examination and five year change in alcohol intake was significant $(\mathrm{P}=0.03)$. Hence, the effect of change in alcohol intake depended on the starting point in 1993-98 and, as a consequence, five year change in alcohol intake was stratified by intake in the 1993-98 examination ( $<7,7-13$, and $\geq 14$ drinks per week; web fig). Women who drank seven to 13 drinks per week in the 1993-98 examination and reduced their intake by 3.6 drinks per week or more had a significantly higher risk of mortality risk than women with a stable alcohol intake (that is, change in alcohol intake of 0 drinks per week; web fig 3B). Likewise, women who drank at least 14 drinks per week in 1993-98 and who increased their intake by 7.1 drinks per week or more had a significantly higher risk of mortality risk than women with a stable alcohol intake (web fig 3C). However, the main part of results from these analyses was ambiguous, probably due to the stratification on alcohol intake in the 1993-98 examination leading to less statistical power.

\section{Discussion}

\section{Principal findings}

Overall, results of this study showed that postmenopausal women who increased their alcohol intake over a five year period had a higher risk of breast cancer and a lower risk of coronary heart disease than those women with a stable alcohol intake. Results among women who reduced their alcohol intake over the five year period were not significantly associated with risk of breast cancer or coronary heart disease. Analyses of all cause mortality showed-as expected-that women who increased their alcohol intake further from a high intake ( $\geq 14$ drinks per week) had a higher mortality risk than women with a stable high intake.

\section{Strengths and limitations of the study}

Strengths of the study included the prospective design minimising risk bias due to reverse causality and a large study size. Limitations included the fact that alcohol intake was self-reported and possibly underestimated, although the method is generally assumed to be valid ${ }^{6}$ and studies comparing self-administered questionnaires with intake assessed by detailed diet records have shown no substantial dissimilarities. ${ }^{35} 36$ In addition, the food frequency questionnaire used in this study was interviewer verified. ${ }^{20}$ Changes in alcohol intake might have occurred at any time between the two examinations, and women reporting a stable intake could have had variations in intake as well. Non-differential misclassification of alcohol intake was likely present at both examinations, leading to more random variation when studying change in alcohol intake compared to studies using alcohol intake at one point in time only, leading to lower effective statistical power.

Concerning the definition and operationalisation of alcohol as exposure, a limitation is that drinking patterns were not taken into account. Studies have shown an adverse effect of alcohol on coronary heart disease and increased risk of death when drinking in binges. ${ }^{10123738}$ Hence, residual confounding from binge drinking could have influenced the results. Breast feeding has consistently been associated with lower risk of breast cancer in epidemiological studies. ${ }^{39}$ Other reproductive factors such as older age at menarche might also associate with risk, possibly depending on the breast cancer subtype ${ }^{40} \mathrm{~A}$ weakness in our study was that we were unable to adjust for reproductive factors other than parity and number of births; if such factors were associated with change in alcohol intake, results could be biased due to residual confounding.

A common issue in observational studies is the risk of bias due to sick quitting. ${ }^{1117}$ However, in this study, we did sensitivity analyses that excluded women with a history of benign breast tumour surgery before baseline from the analyses for breast cancer, and excluded women with any type of cardiovascular disease before baseline from the analyses for coronary heart disease. We also performed analyses in which we omitted the first three years of follow-up, and excluded abstainers, which did not change results.

We used coronary heart disease as an outcome, although the validity of the diagnosis of myocardial infarction is probably higher. In a validation study, 94\% of myocardial infarction cases in the Danish Hospital Discharge Register and the Danish Register of Causes of Death were confirmed. ${ }^{41}$ Presumably, the validity of the diagnosis of coronary heart disease as an entity is lower. ${ }^{34}$ However, we found no differences in results when performing the analyses with myocardial infarction as the outcome instead of coronary heart disease.

A final limitation is that only $37 \%$ of the invited women participated in the first examination in 1993-98, and hence caution should be taken when generalising the findings. Women who chose to participate might have had a different risk profile and be in better health than those who declined to participate. Nonetheless, the studied associations will be biased only if non-response is related to both the exposure (that is, change in alcohol intake) and the outcome of the study (that is, breast cancer or coronary heart disease). Recent studies seem to indicate that non-responders have higher rates of alcohol related mortality and morbidity. ${ }^{42}$ But in this study, the range of variation in the information collected on alcohol and other lifestyle risk factors was similar to previous Danish studies, ${ }^{19}$ and the observed incidences of breast cancer and coronary heart disease did not differ from those of the general population. 


\section{Potential mechanisms}

Biological mechanisms explaining the relation between alcohol intake and risk of breast cancer are complex. One of the most common hypotheses is that alcohol increases breast cancer risk via alterations in circulating oestrogen levels. ${ }^{8943}$ Another hypothesis concerns alcohol metabolism by which alcohol is converted to acetaldehyde by alcohol dehydrogenase (ADH), which, for example, is expressed in the breast tissue. Acetaldehyde is genotoxic and is considered to increase breast cancer risk by several mechanisms, for instance by damaging DNA. ${ }^{238}$ Evidence linking alcohol to a lower risk of coronary heart disease includes alcohol increased levels of high density lipoprotein cholesterol, reduced levels of fibrinogen, and inhibited platelet activation in experimental studies, mechanisms that theoretically contribute to a beneficial effect. ${ }^{10-13}$ However, this evidence is ambiguous. ${ }^{44} 45$ In addition, a large scale Mendelian randomisation study (sometimes referred to as nature's randomised trial) of alcohol intake and coronary heart disease demonstrated that carriers of the A allele of $A D H 1 B$ (rs1229984), a genetic variant associated with non-drinking and lower alcohol consumption, had lower odds of coronary heart disease. ${ }^{45}$ This result might add scepticism to the alleged protective effects of alcohol on the risk of coronary heart disease.

Because the development of breast cancer is assumed to happen over years, ${ }^{8}$ there might be a temporal issue in addressing the effect of a change in alcohol intake over a period of five years only. Nonetheless, a Danish study suggested that recent alcohol intake might be a more important determinant of postmenopausal breast cancer risk than earlier exposure. ${ }^{46}$ Other studies found that alcohol drinking throughout adult life was associated with higher risk of breast cancer, ${ }^{47}$ and that alcohol intake at baseline was more important than alcohol intake at a later time point. ${ }^{48}$ Compared to the results of our study, the effect of change in alcohol intake on the risk of breast cancer and coronary heart disease was independent of alcohol intake at the starting point in the 1993-98 examination. However, we did not take lifetime alcohol consumption into account, and also were unable to account for changes in alcohol intake during follow-up.

\section{Comparison with other studies}

To the best of our knowledge, no previous studies have investigated the association between change in alcohol intake and risk of breast cancer, whereas a few studies, also including women, have addressed the risk of coronary heart disease. Okwuosa and colleagues studied long term changes in alcohol consumption status and variations in fibrinogen levels in a cohort of 2520 young men and women. ${ }^{49}$ Compared with participants who never drank, those who became or stayed drinkers had smaller increases in fibrinogen, while those who quit drinking had the highest increase over 13 years of follow-up.

King and colleagues followed a cohort of 7697 men and women aged 45-64 years who were non-drinkers at baseline over a 10 year period. ${ }^{50}$ Within six years, $6.0 \%$ of participants began moderate alcohol consumption, and $0.4 \%$ began heavier drinking. After four years of follow-up, new moderate drinkers had a $38 \%$ lower risk of cardiovascular disease than the persistently non-drinking participants. In a longitudinal population based study, Grønbaek and researchers studied changes in alcohol intake over a five year period and mortality in 6644 men and 8010 women aged 25-98 years. ${ }^{18}$ For coronary heart disease mortality, stable non-drinkers had a relative risk of 1.32 (95\% confidence interval 0.97 to 1.79) compared with stable light drinkers, those who had decreased their drinking from light to none increased their risk (1.40 (1.00 to 1.95)), and those who had increased from non-drinking to light drinking decreased their risk (0.71 (0.44 to 1.14$)$ ).

In addition, Lazarus and colleagues studied changes in alcohol consumption and risk of death from ischemic heart disease in 1845 men and 2225 women aged 35 years and older. ${ }^{51}$ There was a significantly higher risk of death from ischaemic heart disease in women who gave up drinking between 1965 and 1974 than in women who continued to drink (relative risk 2.75 (95\% confidence interval 1.44 to 5.23)). Furthermore, several studies that only included men have also examined the association between change in alcohol intake and coronary heart disease. ${ }^{52-57}$

\section{Implications}

Our study prepares the ground for future research aimed to clarify whether our observed associations can be seen in other study populations. In addition, more research is needed concerning the biological mechanisms behind the relation between alcohol intake and breast cancer. For instance, this might help to elucidate the influence of time of exposure, which could be an important factor when giving public health advice.

In terms of public health implications of this study, our findings suggest increased risks of breast cancer following an increase in alcohol intake compared to a stable intake, whereas a decreased risk of coronary heart disease was observed. However, in the broader picture, additional analyses on all cause mortality support current recommendations of a light to moderate intake.

\section{Conclusions}

Postmenopausal women who increased their alcohol intake over a five year period between the 1993-98 and 1999-2003 examinations in the Diet, Cancer, and Health Study had a higher risk of breast cancer and a lower risk of coronary heart disease compared with women with a stable alcohol intake. Results among women who decreased their alcohol intake over the five year period were not significantly associated with risk of breast cancer or coronary heart disease. Analyses of all cause mortality indicated that women who increased their alcohol intake further from a high intake (that is, from $\geq 14$ drinks per week) had a higher mortality risk than 
women with a stable high intake. Altogether, our findings support that alcohol is associated with breast cancer (increased risk) and coronary heart disease (decreased risk).

We thank the participants of the Diet, Cancer, and Health Study for their time and cooperation.

Contributors: IST conceived the study idea and is the study guarantor. IST and MKD designed and performed the analyses, and drafted the manuscript. All authors contributed to discussing and commenting on the paper.

Funding: This study received no funding.

Competing interests: All authors have completed the ICMJE uniform disclosure form at www.icmje.org/coi_disclosure.pdf and declare: no support from any organisation for the submitted work; no financial relationships with any organisations that might have an interest in the submitted work in the previous three years; no other relationships or activities that could appear to have influenced the submitted work.

Ethical approval: Not required.

Data sharing: No additional data available.

The guarantor affirms that the manuscript is an honest, accurate, and transparent account of the study being reported; that no important aspects of the study have been omitted; and that any discrepancies from the study as planned have been explained.

This is an Open Access article distributed in accordance with the Creative Commons Attribution Non Commercial (CC BY-NC 3.0) license, which permits others to distribute, remix, adapt, build upon this work non-commercially, and license their derivative works on different terms, provided the original work is properly cited and the use is non-commercial. See: http://creativecommons.org/licenses/ by-nc/3.0/.

1 Anderson P. MLGG. Alcohol in the European Union-consumption, harm and policy approaches. World Health Organization, 2012.

2 Seitz HK, Pelucchi C, Bagnardi V, La Vecchia C. Epidemiology and pathophysiology of alcohol and breast cancer: Update 2012. Alcohol Alcohol 2012;47:204-12. doi:10.1093/alcalc/ags011.

3 Scoccianti C, Lauby-Secretan B, Bello PY, Chajes V, Romieu I. Female breast cancer and alcohol consumption: a review of the literature. $A m$ J Prev Med 2014;46(suppl 1):S16-25. doi:10.1016/j. amepre.2013.10.031.

4 Hamajima N. Hirose K Tajima K et al. Collaborative Group on Hormonal Factors in Breast Cancer. Alcohol, tobacco and breast cancer-collaborative reanalysis of individual data from 53 epidemiological studies, including 58,515 women with breast cancer and 95,067 women without the disease. Br/Cancer 2002;87:1234-45. doi:10.1038/sj.bjc.6600596.

5 Allen NE, Beral V, Casabonne D, et al. Million Women Study Collaborators. Moderate alcohol intake and cancer incidence in women. J Natl Cancer Inst 2009;101:296-305. doi:10.1093/jnci/ djn514.

6 Roerecke M, Rehm J. The cardioprotective association of average alcohol consumption and ischaemic heart disease: a systematic review and meta-analysis. Addiction 2012;107:1246-60. doi:10.1111/j.1360-0443.2012.03780.x.

7 Ronksley PE, Brien SE, Turner BJ. Mukamal KJ, Ghali WA. Association of alcohol consumption with selected cardiovascular disease outcomes: a systematic review and meta-analysis. BMJ 2011;342:d671 doi:10.1136/bmj.d671.

8 Brooks PJ, Zakhari S. Moderate alcohol consumption and breast cancer in women: from epidemiology to mechanisms and interventions. Alcohol Clin Exp Res 2013;37:23-30. doi:10.1111/j.1530-0277.2012.01888.x.

9 Dorgan JF, Baer DJ, Albert PS, et al. Serum hormones and the alcohol-breast cancer association in postmenopausal women. J Natl Cancer Inst 2001:93:710-5. doi:10.1093/inci/93.9.710.

10 Rehm J, Baliunas D, Borges GL, et al. The relation between different dimensions of alcohol consumption and burden of disease: an overview. Addiction 2010;105:817-43. doi:10.1111/i.1360-0443.2010.02899x

11 Emberson JR, Bennett DA. Effect of alcohol on risk of coronary heart disease and stroke: causality, bias, or a bit of both?Vasc Health Risk Manag 2006:2:239-49. doi:10.2147/vhrm.2006.2.3.239.

12 Hill JA. In vino veritas: alcohol and heart disease. Am J Med Sci 2005;329:124-35. doi:10.1097/00000441-200503000-00003.

13 Brien SE, Ronksley PE, Turner BJ, Mukamal KJ, Ghali WA. Effect of alcohol consumption on biological markers associated with risk of coronary heart disease: systematic review and metaanalysis of interventional studies. BMJ 2011;342:d636 doi:10.1136/bmi.d636.
14 Wang C, Xue H, Wang Q, et al. Effect of drinking on all-cause mortality in women compared with men: a meta-analysis. J Womens Health (Larchmt) 2014:23:373-81. doi:10.1089/jwh.2013.4414.

15 Shekelle P. Review: high alcohol intake increases mortality in both men and women. ACP J Club 2007;146:48.

16 Di Castelnuovo A, Costanzo S, Bagnardi V, Donati MB, lacoviello L, de Gaetano G. Alcohol dosing and total mortality in men and women: an updated meta-analysis of 34 prospective studies. Arch Intern Med 2006;166:2437-45. doi:10.1001/archinte.166.22.2437.

17 Rehm J, Roerecke M. Reduction of drinking in problem drinkers and all-cause mortality. Alcohol Alcohol 2013;48:509-13. doi:10.1093/ alcalc/agt021.

18 Grønbaek M, Johansen D, Becker U, et al. Changes in alcohol intake and mortality: a longitudinal population-based study. Epidemiology 2004;15:222-8. doi:10.1097/01.ede.0000112219.01955.56.

19 Tjønneland A, Olsen A, Boll K, et al. Study design, exposure variables, and socioeconomic determinants of participation in Diet, Cancer and Health: a population-based prospective cohort study of 57,053 men and women in Denmark. Scand J Public Health 2007;35:432-41. doi:10.1080/14034940601047986.

20 Tjønneland A, Overvad K, Haraldsdóttir J, Bang S, Ewertz M, Jensen $\mathrm{OM}$. Validation of a semiquantitative food frequency questionnaire developed in Denmark. Int J Epidemiol 1991;20:906-12. doi:10.1093/ ije/20.4.906.

21 Overvad K, Tjønneland A, Haraldsdóttir J, Ewertz M, Jensen OM. Development of a semiquantitative food frequency questionnaire to assess food, energy and nutrient intake in Denmark. Int J Epidemiol 1991;20:900-5. doi:10.1093/ije/20.4.900.

22 Trichopoulou A, Orfanos P, Norat T, et al. Modified Mediterranean diet and survival: EPIC-elderly prospective cohort study. BMJ 2005:330:991. doi:10.1136/bmj.38415.644155.8F

23 Storm HH, Michelsen EV, Clemmensen IH, Pihl J. The Danish Cancer Registry--history, content, quality and use. Dan Med Bull 1997:44:535-9.

24 Storm HH. Completeness of cancer registration in Denmark 1943-1966 and efficacy of record linkage procedures. Int J Epidemiol 1988;17:44-9. doi:10.1093/ije/17.1.44.

25 Jürgensen HJ, Frølund C, Gustafsen J, Mosbech H, Guldhammer B, Mosbech J. Registration of diagnoses in the Danish National Registry of Patients. Methods Inf Med 1986;25:158-64.

26 Helweg-Larsen K. The Danish Register of Causes of Death. Scand I Public Health 2011;39(Suppl):26-9. doi:10.1177/1403494811399958.

27 Juel K, Helweg-Larsen K. The Danish registers of causes of death. Dan Med Bull 1999;46:354-7.

28 Jensen AR, Overgaard J, Storm HH. Validity of breast cancer in the Danish Cancer Registry. A study based on clinical records from one county in Denmark. Eur J Cancer Prev 2002;11:359-64. doi:10.1097/00008469-200208000-00007.

29 Mosbech J, Jørgensen J, Madsen M, Rostgaard K, Thornberg K, Poulsen TD. [The national patient registry. Evaluation of data quality]. Ugeskr Laeger 1995;157:3741-5.

30 Spratt M, Carpenter J, Sterne JA, et al. Strategies for multiple imputation in longitudinal studies. Am J Epidemiol 2010;172:478-87. doi:10.1093/aje/kwq137.

31 Rubin DB. Multiple imputation for nonresponse in surveys.John Wiley and Sons, 2004

32 Greenland S. Dose-response and trend analysis in epidemiology: alternatives to categorical analysis. Epidemiology 1995;6:356-65. doi:10.1097/00001648-199507000-00005

33 Harrell J, Frank E.Relaxing linearity assumption for continuous predictors. Regression modeling strategies with applications to linear models, logistic regression, and survival analysis.Springer Science+Business Media New York, 2001.

34 Joensen AM, Jensen MK, Overvad K, et al. Predictive values of acute coronary syndrome discharge diagnoses differed in the Danish National Patient Registry. J Clin Epidemiol 2009;62:188-94. doi:10.1016/j.jclinepi.2008.03.005.

35 Grønbaek M, Heitmann BL. Validity of self-reported intakes of wine, beer and spirits in population studies. Eur J Clin Nutr 1996;50:487-90

36 Giovannucci E, Colditz G, Stampfer MJ, et al. The assessment of alcohol consumption by a simple self-administered questionnaire. Am J Epidemiol 1991;133:810-7.

37 Bagnardi V, Zatonski W, Scotti L, La Vecchia C, Corrao G. Does drinking pattern modify the effect of alcohol on the risk of coronary heart disease? Evidence from a meta-analysis. J Epidemiol Community Health 2008;62:615-9. doi:10.1136/ jech.2007.065607.

38 Roerecke M, Rehm J. Irregular heavy drinking occasions and risk of ischemic heart disease: a systematic review and meta-analysis. Am J Epidemiol 2010;171:633-44. doi:10.1093/aje/kwp451.

39 Hanf V, Hanf D. Reproduction and breast cancer risk. Breast Care (Basel) 2014;9:398-405. doi:10.1159/000369570.

40 Anderson KN, Schwab RB, Martinez ME. Reproductive risk factors and breast cancer subtypes: a review of the literature. Breast Cancer Res Treat 2014:144:1-10. doi:10.1007/s10549-014-2852-7. 
41 Madsen M, Davidsen M, Rasmussen S, Abildstrom SZ, Osler M. The validity of the diagnosis of acute myocardial infarction in routine statistics: a comparison of mortality and hospital discharge data with the Danish MONICA registry. J Clin Epidemiol 2003;56:124-30 doi:10.1016/S0895-4356(02)00591-7.

42 Christensen Al, Ekholm O Gray L, Glümer C, Juel K. What is wrong with non-respondents? Alcohol-, drug- and smoking-related mortality and morbidity in a 12-year follow-up study of respondents and non-respondents in the Danish Health and Morbidity Survey. Addiction 2015:110:1505-12 doi:10.1111/add.12939.

43 Vatsalya V, Issa JE, Hommer DW, Ramchandani VA. Pharmacodynamic effects of intravenous alcohol on hepatic and gonadal hormones: influence of age and sex. Alcohol Clin Exp Res 2012;36:207-13. doi:10.1111/j.1530-0277.2011.01600.x

44 Chikritzhs T, Stockwell T, Naimi T, Andreasson S, Dangardt F, Liang W. Has the leaning tower of presumed health benefits from 'moderate' alcohol use finally collapsed?Addiction 2015;110:726-7. doi:10.1111/ add.12828.

45 Holmes MV, Dale CE, Zuccolo L, et al. InterAct Consortium. Association between alcohol and cardiovascular disease: Mendelian randomisation analysis based on individual participant data. BMJ 2014;349:g4164. doi:10.1136/bmj.g4164.

46 Tjønneland A, Christensen J, Thomsen BL, et al. Lifetime alcohol consumption and postmenopausal breast cancer rate in Denmark: a prospective cohort study. J Nutr 2004;134:173-8

47 Chen WY, Rosner B, Hankinson SE, Colditz GA, Willett WC. Moderate alcohol consumption during adult life, drinking patterns, and breast cancer risk. JAMA 2011;306:1884-90. doi:10.1001/jama.2011.1590.

48 Thygesen LC, Mørch LS, Keiding N, Johansen C, Grønbaek M. Use of baseline and updated information on alcohol intake on risk for breast cancer: importance of latency. Int J Epidemiol 2008;37:669-77. doi:10.1093/ije/dyn060.

49 Okwuosa TM, Klein O, Chan C, Schreiner P, Liu K, Green D. Long-term change in alcohol-consumption status and variations in fibrinogen levels: the coronary artery risk development in young adults (CARDIA) study. BMJ Open 2013;3:e002944. doi:10.1136/ bmjopen-2013-002944.

50 King DE, Mainous AG 3rd, , Geesey ME. Adopting moderate alcohol consumption in middle age: subsequent cardiovascular events. Am J Med 2008;121:201-6. doi:10.1016/j.amjmed.2007.12.004.
51 Lazarus NB, Kaplan GA, Cohen RD, Leu DJ. Change in alcoho consumption and risk of death from all causes and from ischaemic heart disease. BM/1991:303:553-6. doi:10.1136/bmi.303.6802.553.

52 Britton A, Marmot MG, Shipley MJ. How does variability in alcohol consumption over time affect the relationship with mortality and coronary heart disease?Addiction 2010;105:639-45. doi:10.1111/j.1360-0443.2009.02832.x.

53 Emberson JR, Shaper AG, Wannamethee SG, Morris RW, Whincup PH. Alcohol intake in middle age and risk of cardiovascular disease and mortality: accounting for intake variation over time. Am J Epidemio 2005;161:856-63. doi:10.1093/aje/kwi111.

54 Wellmann J, Heidrich J, Berger K, Döring A, Heuschmann PU, Keil U. Changes in alcohol intake and risk of coronary heart disease and all-cause mortality in the MONICA/KORA-Augsburg cohort 1987-97. Eur J Cardiovasc Prev Rehabil 2004;11:48-55. doi:10.1097/01. hjr.0000118174.70522.20.

55 Mukamal KJ, Conigrave KM, Mittleman MA, et al. Roles of drinking pattern and type of alcohol consumed in coronary heart disease in men. N Engl / Med 2003;348:109-18. doi:10.1056/ NEJMoa022095.

56 Wannamethee SG, Shaper AG. Taking up regular drinking in middle age: effect on major coronary heart disease events and mortality. Heart 2002;87:32-6. doi:10.1136/heart.87.1.32.

57 Sesso HD, Stampfer MJ, Rosner B, Hennekens CH, Manson JE, Gaziano JM. Seven-year changes in alcohol consumption and subsequent risk of cardiovascular disease in men. Arch Intern Med 2000;160:2605-12. doi:10.1001/archinte.160.17.2605.

(c) BMJ Publishing Group Ltd 2016

Web table: Hazard ratios (95\% confidence intervals) of all-cause mortality by alcohol intake in the 1993-1998 and 1999-2003 examinations

Web figure: Hazard ratios of all cause mortality by 5 -year change in alcohol intake for women drinking $<7$ (3a), 7-13 (3b), and 14+ (3c) drinks per week, respectively, in the 1993-1998 examination 